\title{
A second-order accurate lattice Boltzmann non-Newtonian flow model
}

\author{
J Boyd $^{1,2}$, J Buick ${ }^{1,2}$ and S Green ${ }^{2}$ \\ ${ }^{1}$ Physics and Electronics, University of New England, Armidale, NSW, 2351, Australia \\ ${ }^{2}$ Cardiovascular Research Group, University of New England, Armidale, NSW, 2351, Australia \\ E-mail: jboyd3@pobox.une.edu.au
}

Received 2 June 2006, in final form 9 October 2006

Published 1 November 2006

Online at stacks.iop.org/JPhysA/39/14241

\begin{abstract}
A second-order accurate lattice Boltzmann model is presented for nonNewtonian flow. The non-Newtonian nature of the flow is implemented using a power law model. This is used to enable the accuracy of the model to be assessed and is not a limitation of the model. The second-order accuracy is demonstrated for a range of power law model parameter values representing shear thinning and shear thickening fluids. These results are compared with those of Gabbanelli et al (2006 Phys. Rev. E 72 046312) and it is noted that a higher order of accuracy and greater computational efficiency are achieved. These results demonstrate the suitability of the LBM for shear-dependent nonNewtonian flow simulations.
\end{abstract}

PACS numbers: 47.11.-j, 47.11.Qr, 47.50.-d

\section{Introduction}

The study of non-Newtonian fluids and their flow properties is of interest in many fields of research, including geophysics (Ashby and Verrall 1977), hydrology (Federico 1998), material sciences (Bird and West 1995) and physiological modelling (Nichols and O'Rourke 2005). In many cases of interest, analytic solutions do not exist due to complex geometries and nonNewtonian properties of the flow. Thus numerical simulation becomes a valuable investigative tool.

In this paper we will consider a lattice Boltzmann method (LBM) for the simulation of non-Newtonian fluids. The LBM is a second-order accurate method for simulating fluid flow that uses a simplified kinetic equation. It has been applied to many general problems including turbulence (Cosgrove et al 2003), magnetohydrodynamics (Chen et al 1991), porous media flow (Manz et al 1999) multiphase flows (Swift et al 1996) and blood flow (Fang et al 2002, Tamagawa and Matsuo 2004, Artoli et al 2004, Boyd et al 2005, Yi et al 2005) as well as to 


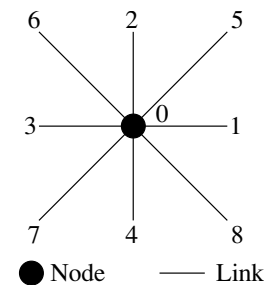

Figure 1. The D2Q9 lattice. The black circle is the node, and the lines are the link directions, numbered from 1 to 8 .

non-Newtonian flows (Gabbanelli et al 2006). The underlying lattice structure and the local nature of the calculation makes it ideal for parallel implementation (Kandhai et al 1998).

In this paper, a non-Newtonian fluid flow model simulated using the LBM will be described and its accuracy will be demonstrated in a two-dimensional rigid pipe flow. A power law model is used to describe the non-Newtonian nature of the fluid. This model is used to enable comparison of the results with an analytic solution, but is not a restriction of the technique. It is found that the LBM retains second-order accuracy for this non-Newtonian flow, demonstrating the suitability of the LBM for shear-dependent non-Newtonian flow simulations, including those that involve more complicated constitutive fluid descriptions.

\section{Theoretical background}

\subsection{The lattice Boltzmann method}

The lattice Boltzmann method (Chen and Doolen 1998) has recently been developed as an alternative method for simulating a range of fluid flows. In the LBM particle distribution functions, $f_{i}(\mathbf{x}, t)$ at point $\mathbf{x}$ at time $t$, are confined to move synchronously on a regular lattice. The distribution functions interact on the lattice in a way that conserves mass, momentum, isotropy and Galilean invariance. Here $i$ labels the lattice link the distribution function is on. The lattice used in this paper is the D2Q9, shown in figure 1.

The evolution of the distribution functions on the lattice is governed by the discrete Boltzmann equation (Chen and Doolen 1998)

$$
f_{i}\left(\mathbf{x}+\mathbf{e}_{i} \Delta x, t+\Delta t\right)=f_{i}(\mathbf{x}, t)+\Omega_{i}(\mathbf{x}, t), \quad(i=0,1, \ldots, M),
$$

where for the D2Q9 lattice, see figure 1,

$$
\begin{array}{ll}
\mathbf{e}_{0}=(0,0), & (i=0), \\
\mathbf{e}_{i}=\left(\cos \left(\frac{\pi}{2}(i-1)\right), \sin \left(\frac{\pi}{2}(i-1)\right)\right), & (i=1,2,3,4), \\
\mathbf{e}_{i}=\sqrt{2}\left(\cos \left(\frac{\pi}{2}(i-1)+\frac{\pi}{4}\right), \sin \left(\frac{\pi}{2}(i-1)+\frac{\pi}{4}\right)\right), & (i=5,6,7,8),
\end{array}
$$

and $\Omega_{i}$ is the collision operator. The fluid density $\rho$ and velocity $\mathbf{u}$ can be calculated directly from the distribution functions at each node by

$$
\rho=\sum_{i} f_{i} \quad \text { and } \quad \rho \mathbf{u}=\sum_{i} f_{i} \mathbf{e}_{i}
$$

It is assumed that the distribution functions $f_{i}$ can be expanded formally around a local equilibrium distribution such that

$$
f_{i}=f_{i}^{\mathrm{eq}}+\varepsilon f_{i}^{\text {neq }},
$$


where $\varepsilon$ is a small parameter often taken to be the Knudson number, $f_{i}^{\text {eq }}$ are equilibrium distribution function and $f_{i}^{\text {neq }}$ are non-equilibrium distributions functions. $f_{i}^{\text {eq }}$ is selected such that

$$
\rho=\sum_{i} f_{i}^{\mathrm{eq}} \quad \text { and } \quad \rho \mathbf{u}=\sum_{i} f_{i}^{\mathrm{eq}} \mathbf{e}_{i}
$$

and it is assumed that the non-equilibrium distribution functions, $f_{i}^{\text {neq }}$, can be further expanded as

$$
f_{i}^{\text {neq }}=f_{i}^{(1)}+\varepsilon f_{i}^{(2)}+o\left(\varepsilon^{2}\right)
$$

where

$$
\sum_{i} f_{i}^{(k)}=\sum_{i} f_{i}^{(k)} \mathbf{e}_{i}=0, \quad k=1,2
$$

The collision operator $\Omega_{i}$ is given by the Bhatnagar-Gross-Krook approximation as (Bhatnagar et al 1954, Chen and Doolen 1998)

$$
\Omega_{i}=\frac{-1}{\tau}\left[f_{i}(\mathbf{x}, t)-f_{i}^{\mathrm{eq}}(\mathbf{x}, t)\right]
$$

where $\tau$ is the relaxation time. The equilibrium form of the distribution function in two dimensions for the D2Q9 lattice is given by (Quian et al 1992)

$$
f_{i}^{\mathrm{eq}}(\mathbf{x}, t)=w_{i} \rho\left(1+3 \mathbf{e}_{i} \cdot \mathbf{u}+\frac{9}{2}\left(\mathbf{e}_{i} \cdot \mathbf{u}\right)^{2}-\frac{3}{2} \mathbf{u}^{2}\right)
$$

where $w_{0}=4 / 9, w_{i}=1 / 9$ for $i=1,2,3,4$ and $w_{i}=1 / 36$ for $i=5,6,7,8$. The relaxation time $\tau$ is related to the kinematic viscosity $v$ by

$$
v=\frac{2 \tau-1}{6}
$$

The LBM reproduces the Navier stokes equation in the nearly incompressible limit and is second-order accurate in the body of the fluid (Chen and Doolen 1998).

The stress tensor for an incompressible fluid with pressure $p$ is given by

$$
\sigma_{\alpha \beta}=-p \delta_{\alpha \beta}+2 \eta S_{\alpha \beta}
$$

where $\delta_{\alpha \beta}$ is the Kronecker delta and

$$
S_{\alpha \beta}=\frac{1}{2}\left(\nabla_{\beta} u_{\alpha}+\nabla_{\alpha} u_{\beta}\right)
$$

is the strain rate tensor.

It can be shown (Artoli 2003) that $S_{\alpha \beta}$ can be calculated locally at each node in the LBM as

$$
S_{\alpha \beta}=-\frac{3}{2 \tau} \sum_{i} f_{i}^{(1)} \mathbf{e}_{i \alpha} \mathbf{e}_{i \beta} .
$$

The $f_{i}^{(1)}$ terms are usually calculated as part of the velocity calculations in the LBM algorithm. Thus calculating shear in this manner is efficient since it removes the need to calculate derivatives of the velocity. Further, the shear is calculated locally, which is particularly advantageous if the LBM is being implemented in parallel. 


\subsection{The power law model}

In the following discussion we denote the second invariant of the strain rate tensor as

$$
D_{I I}=\sum_{\alpha, \beta=1}^{l} S_{\alpha \beta} S_{\alpha \beta},
$$

where $l=2$ in the case of a two-dimensional model. The shear rate is then defined as

$$
\dot{\gamma}=2 \sqrt{D_{I I}} \text {. }
$$

The power law model is one of the simplest generalizations of non-Newtonian flow. In this model the apparent viscosity is given by (Quarteroni et al 2000)

$$
v(\dot{\gamma})=m|\dot{\gamma}|^{n-1},
$$

where $m$ and $n$ are parameters that are usually obtained by fitting equation (16) to physical viscometric data. This model has the following analytic solution for a simple steady flow in a rigid two-dimensional pipe (Robson 2003):

$$
u(y)=\left(\frac{G}{2 m}\right)^{\frac{1}{n}}\left(\frac{n}{n+1}\right)\left[\left(\frac{L}{2}\right)^{\frac{n+1}{n}}-\left(\left|\frac{L}{2}-y\right|\right)^{\frac{n+1}{n}}\right],
$$

where $L$ is the pipe diameter and $G=-\frac{\mathrm{d} p}{\mathrm{~d} x}$ is the pressure gradient driving the flow.

The value of the parameter $n$ determines the response of the fluid to changes in shear rate, for $n<1$ the fluid is shear thinning, for $n=1$ the fluid is Newtonian and for $n>1$ the fluid is shear thickening.

We note that for a shear thinning fluid (i.e. $n<1$ ), $\lim _{\dot{\gamma} \rightarrow 0} \quad m|\dot{\gamma}|^{n-1}=\infty$. Also, the units of $m$ are $m^{2} s^{n-2}$, and thus this parameter cannot be associated with any physical properties of the fluid.

Equation (16) can be non-dimensionalized to produce the following dimensionless number analogous to the Reynold's number:

$$
\operatorname{Re}_{P L}=\frac{u_{0}^{2-n} L^{n}}{m}
$$

where $m$ and $n$ are the power law parameters and $u_{0}$ is the maximum velocity in a pipe of width $L$.

\section{Methods and results}

Power law non-Newtonian flow was implemented in the LBM through the coupling of equations (10) and (16), giving a shear-dependent relaxation time $\tau$ at each node. Calculation of shear stress, and hence shear strain, was done via equation (13). Simulations were run at $R e_{P L}=100$ for $n$ parameters of $n=0.25,0.5,0.75,1.0$ and 1.25 in order to test the LBM accuracy for a range of non-Newtonian behaviours. A sub-grid accurate second-order boundary scheme (Guo et al 2002) was used to implement the pipe geometry.

$G, L$ and $m$ were varied in the simulations in a manner that ensured the Mach number was $<0.03$ in order to avoid incompressibility artefacts. The simulations were run until the following criterion was satisfied

$$
\sum_{\mathbf{x}}\|\mathbf{u}(\mathbf{x}, t)-\mathbf{u}(\mathbf{x}, t-1)\|<\hat{\varepsilon}
$$

where $\hat{\varepsilon}$ was a small number taken to be $\hat{\varepsilon}=1 \times 10^{-10}$. 


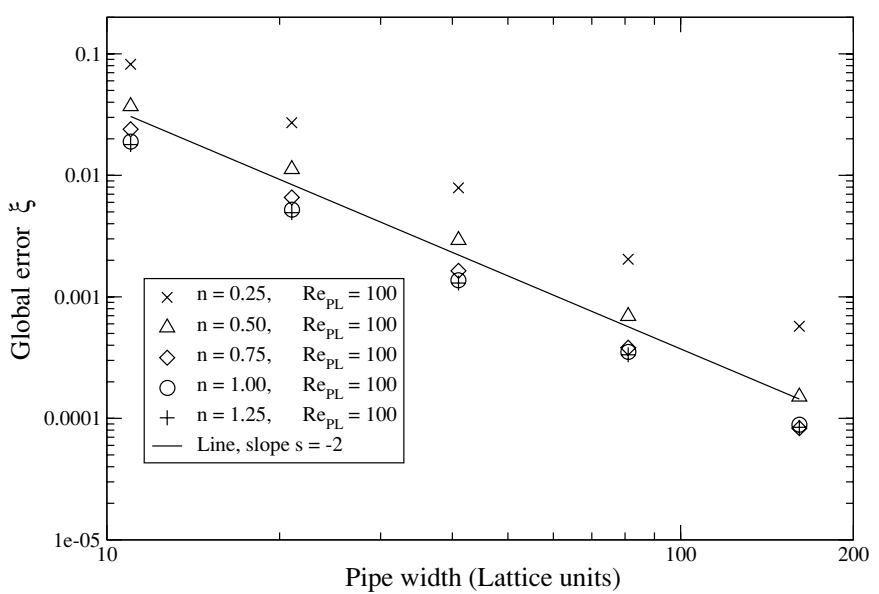

Figure 2. Global error for power law model flow. The black line represents a line of slope -2 , indicating second-order behaviour.

The solutions obtained were compared to the analytic solution given in equation (17), and the global error,

$$
\xi=\frac{\sum_{\mathbf{x}}\left\|\mathbf{u}_{b}(\mathbf{y})-\mathbf{u}_{a}(\mathbf{y})\right\|}{\sum_{\mathbf{x}}\left\|\mathbf{u}_{a}(\mathbf{y})\right\|}
$$

was calculated, where the subscript $a$ refers to the exact analytic solution and the subscript $b$ refers to the simulated Boltzmann value. Results for the global error are shown in figure 2.

The black line in figure 2 represents a line of slope -2 , indicating second-order behaviour. It is observed that the data presented for the different $n$ parameters closely match the slope of this line. Larger errors occur for smaller $n$ values.

Figures $3(a)-(d)$ show the normalized analytic velocity profiles (solid lines) compared to the LBM results (circles) for parameter values $(a) n=0.25,(b) n=0.50,(c) n=0.75$ and (d) $n=1.25$. It can be seen that the LBM accurately simulates the correct velocity profile for both shear thinning $(n<1)$ and shear thickening $(n>1)$ flows.

For $n<1$ we see a general flattening of the velocity profile, with flatter velocity profiles corresponding to lower $n$. Conversely, the profile for $n=1.25$ shows greater curvature near the central peak velocity. This phenomena can be seen more clearly in figure 4 , which shows a comparison of the normalized flow profiles over the range of $n$ parameter values.

\section{Discussion}

Figure 2 shows that the LBM retains second-order accuracy in the case of a two-dimensional power law flow through a rigid pipe. This method is an improvement over the approach of Gabbanelli et al (2006), who used a first-order finite difference method (Aharanov and Rothman 1993) to estimate shear, obtaining only first-order accurate results. The model presented here represents an improvement to a second-order accurate model and an increase in the computational efficiency of the algorithm.

The simulated velocity profiles shown in figure 3 closely match the profiles of the analytic solution for all parameters of $n$. The largest difference can be seen for $n=0.25$, where the 

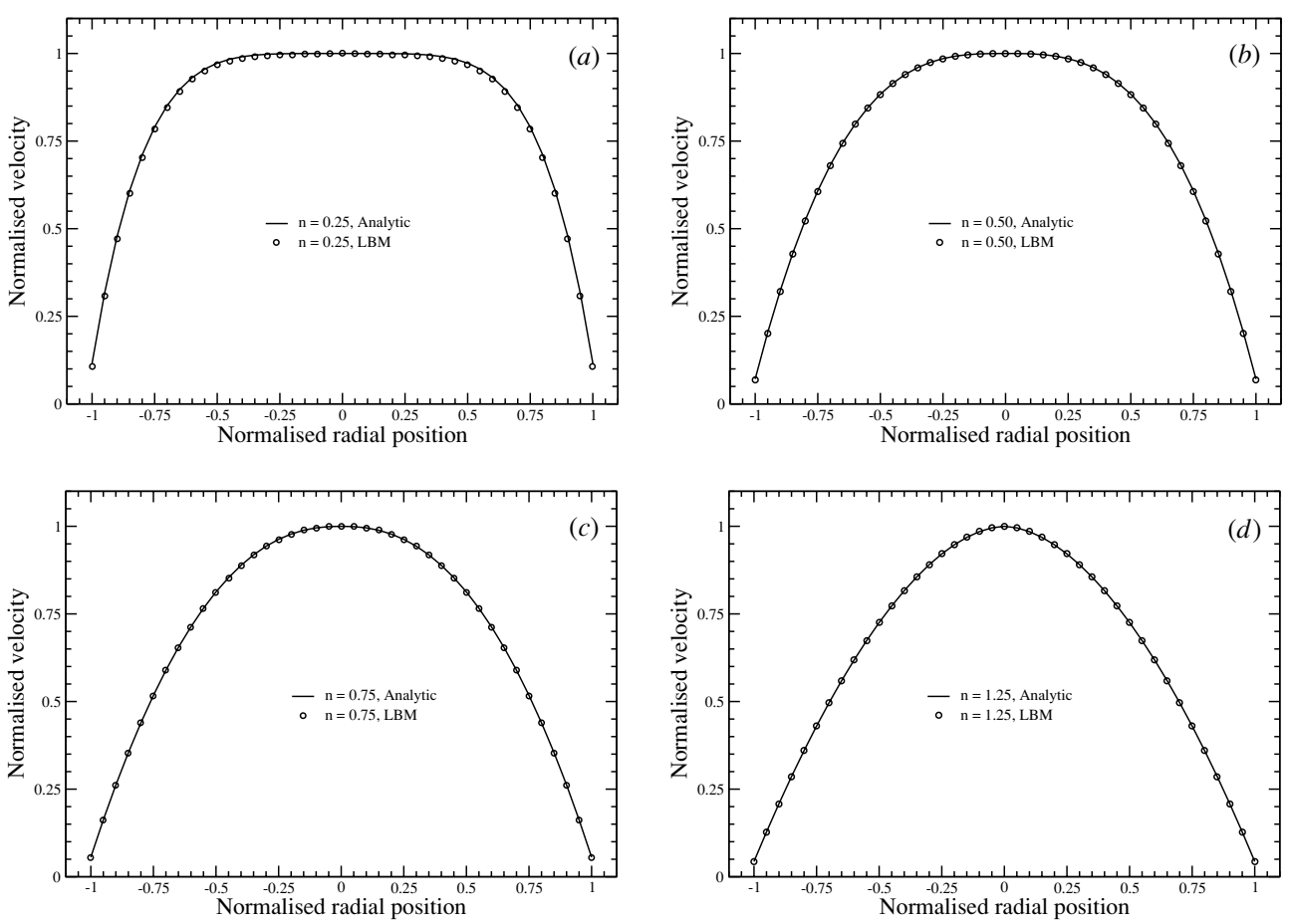

Figure 3. Velocity profile comparisons between the LBM and analytic solutions for power law model flows corresponding to parameter values of $(a) n=0.25$, (b) $n=0.50$, (c) $n=0.75$ and (d) $n=1.25$.

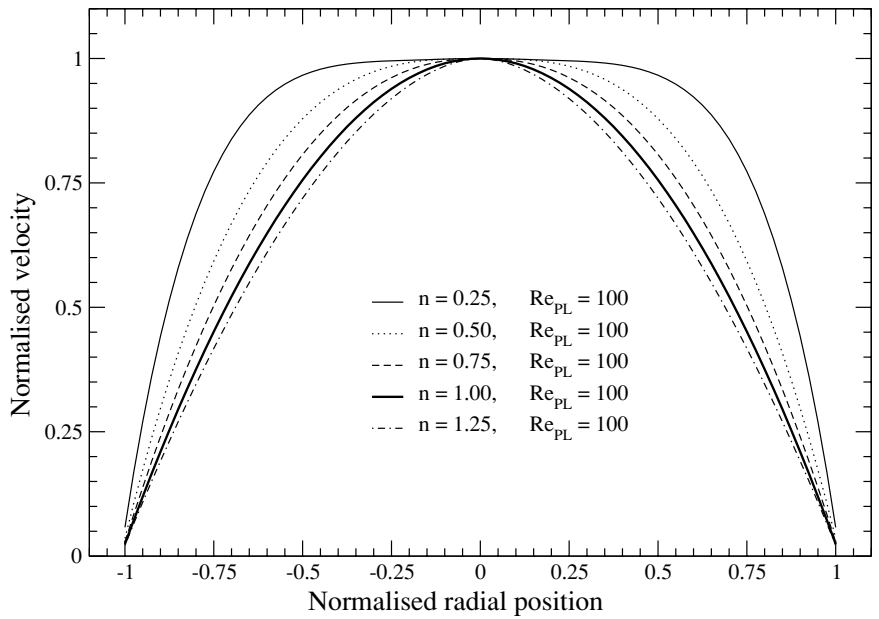

Figure 4. Normalized power law model flow velocity profiles. The thick black line indicates a standard Newtonian parabolic profile.

LBM profile shows slightly less flattening around the central peak velocity compared to the analytic solution. 


\section{Conclusion}

A second-order accurate LBM for shear-dependent non-Newtonian flow has been proposed. This method avoids time consuming derivations of the velocity data to calculate the shear. These results indicate that the LBM is suitable for simulating shear-dependent non-Newtonian flows, including fluids with more complicated constitutive equations.

\section{Acknowledgments}

This work was partially supported by Sigma Xi Grant no 10040015 and the Australian Postgraduate Award (APA); this assistance is gratefully acknowledged.

\section{References}

Aharanov E and Rothman D H 1993 Non-Newtonian flow (through porous media): a lattice-Boltzmann method Geophys. Res. Lett. 20 679-82

Artoli A 2003 Mesoscopic Computational Haemodynamics (Wageningen: Ponsen \& Looijen)

Artoli A M, Kandhai D, Hoefsloot H C J, Hoekstra A G and Sloop P M A 2004 Lattice BGK simulations of flow in a symmetric bifurcation Future Gener. Comput. Syst. 20 909-16

Ashby M F and Verrall R A 1977 Michromechanisms of flow and fracture, and their relevance to the rheology of the upper mantle Phil. Trans. R. Soc. A 288 59-95

Bhatnagar P L, Gross E P and Krook M 1954 A model for collision processes in gases: I. Small amplitude processes in charged and neutral one-component system Phys. Rev. 94 511-25

Bird R B and West J M 1995 Constitutive equations for polymeric liquids Annu. Rev. Fluid. Mech. 27 169-93

Boyd J, Buick J, Cosgrove J A and Stansell P 2005 Application of the lattice Boltzmann model to simulated stenosis growth in a two-dimensional carotid artery Phys. Med. Biol 50 4783-96

Chen S, Chen H, Martinez D and Matthaues W 1991 Lattice Boltzmann model for simulation of magnetohydrodynamics Phys. Rev. Lett. 67 3776-9

Chen S and Doolen G D 1998 Lattice Boltzmann method for fluid flows Annu. Rev. Fluid Mech. 30 329-64

Cosgrove J A, Buick J M, Tonge S J, Munro C G, Greated C A and Campbell D M 2003 Application of the lattice Boltzmann method to transition in oscillatory channel flow J. Phys. A: Math. Gen. 36 2609-20

Fang H P, Wang Z W, Lin Z F and Liu M R 2002 Lattice Boltzmann method for simulating the viscous flow in large distensible blood vessels Phys. Rev. E 65051925

Federico V D 1998 Non-Newtonian Flow in a variable aperture fracture Transport Porous Med. 30 75-86

Gabbanelli S G, Drazer G and Koplik J 2006 Lattice Boltzmann method for non-Newtonian fluid flows Phys. Rev. E 72046312

Guo Z, Zheng C and Shi B 2002 An extrapolation method for boundary conditions in lattice Boltzmann method Phys. Fluids 14 2007-10

Kandhai D, Koponen A, Koekstra A G, Kataja M, Timonen J and Sloot P M A 1998 Lattice-Boltzmann hydrodynamics on parallel systems Comput. Phys. Commun. 111 14-26

Manz B, Gladden L F and Warren P B 1999 Flow and dispersion in porous media: latice-Boltzmann and NMR studies AIChE J. 45 1845-54

Nichols W M and O'Rourke M F 2005 McDonald's Blood Flow in Arteries Theoretical, Experimental and Clinical Principles 5th edn (New York: Oxford University Press)

Quarteroni A, Tuveri M and Veneziani A 2000 Computational vascular fluid dynamics: problems, models and methods Comput. Visual Sci. 2 163-97

Quian Y H, d'Humieres D and Lallemand P 1992 Lattice BGK models for Navier-Stokes equation Europhys. Lett. 17 479-84

Robson J A 2003 A finite element approximation of non-Newtonian flow PhD Thesis University of Manchester

Swift M R, Orlandini W, Osborn W R and Yeomans J M 1996 Lattice Boltzmann simulations of liquid-gas and binary fluid systems Phys. Rev. E 54 5041-52

Tamagawa M and Matsuo S 2004 Predictions of thrombus formation using lattice Boltzmann method-(Modeling of adhesion force for particles to wall) JSME Int. J. C 47 1027-34

Yi H H, Xu S X, Qian Y H and Fang H P 2005 Lattice Boltzmann simulation of blood flow in blood vessels with the rolling massage Chin. Phys. Lett 22 3210-13 